Revista de Psicología de la PUCP. Vol. XXI, 1, 2003 Edición de aniversario

\title{
Orientaciones innovativas en la evaluación y educación de niños en situación de desventaja sociocultural
}

\author{
Alegría Majluf ${ }^{1}$ \\ Universidad Peruana Cayetano Heredia
}

En el presente trabajo se describen los supuestos de la psicometría tradicional, no apropiada para niños que sufren de privación socio-cultural y las nuevas estrategias para la evaluación y educación de estos niños. Para una mejor comprensión de éstas se mencionará la orientación socio-cultural iniciada por Vygotsky, se destacara el concepto de Zona de Desarrollo Próximo, la evaluación Dinámica y el Aprendizaje Mediado, ampliado por Feuerstein y complementado por Coll respecto al Aprendizaje Significativo.

Palabras clave: evaluación, educación, niños, desventaja socio-cultural, aprendizaje significativo, Feuerstein, Vygotsky, Coll.

New approaches for the asessment and education of children under socio-cultural disadvantages

The present paper describes the traditional psychometric parameters that don't fit by working with children who suffer socio-cultural deprivation (or who are living under socio-cultural disadvantages) and the new assessment and education strategies for this population. Vygotsky's socio-cultural approach, that emphasizes the Zone of Proximal Development, and the Dynamic Assessment and the Mediated Learning developed by Feuerstein and complemented by Coll related with the Significative Learning are stressed.

Key words: Assessment, education, children, socio-cultural deprivation, significative learning, Feuerstein, Vygotsky, Coll.

1 Doctora en psicología por el Californian School of Professional Psychology de San Francisco, California. Estudió en la Universidad Nacional Mayor de San Marcos (Perú) la especialidad de Filosofía y Psicología. Fue reconocida como la psicóloga del año en 1994. Actualmente se dedica a la investigación del tema técnicas de crianza-estilo autoritario y democrático. Asimismo, trabaja como psicóloga de la Escuela "Esperanza y Caridad», institución financiada por un grupo de personas de Lima, en donde investiga la relación entre el desarrollo intelectual y la nutrición. 

Tradicionalmente se consideraba que las pruebas psicológicas de inteligencia, las escalas Wechsler: WISC-III, Stanford, Binet, McCarthy eran validas y confiables ya que proporcionaban información útil respecto al niño promedio en los países donde habían sido estandarizadas. Estas pruebas permitían conocer la capacidad intelectual del niño y su perfil de rendimiento. El valor de estas no debe sorprender ya que han sido elaboradas considerando como requisitos indispensables para su uso las siguientes suposiciones:

1. Las experiencias de los niños son semejantes.

2. Las oportunidades de aprendizaje en el hogar y la comunidad son comparables.

3. Los niños tienen igual facilidad con el lenguaje para comprender y seguir las instrucciones.

4. La sintaxis y el uso de vocabulario son familiares al niño.

En los Estados Unidos, país de donde proviene la mayor parte de la tecnología psicométrica y donde se han estandarizado la mayoría de las pruebas, se encuentra, sin embargo, una desviación estándar o más entre los niños blancos americanos y los niños negros, hispanos o indios. Tal diferencia se interpretaba, aun desde la época de Terman (quien elaboró la prueba de Stanford-Binet) como que los niños de la minoría eran inferiores. Tal concepto determinó que el sistema escolar californiano evaluara a niños negros y los ubicara en clases especiales de acuerdo a los resultados de test de Stanford-Binet.

Ante esta situación, muchos psicólogos, entre los 60 y 70, protestaron y decidieron afrontar el problema seleccionando a 6 niños negros ubicados en clases para niños retardados mentales (RM). Los evaluaron cuidadosamente y encontraron que ninguno de ellos era un 
RM, y que habían sido privados de sus derechos civiles en base a resultados de pruebas que no reflejaban su verdadero potencial.

Por ello, en países multiculturales como EEUU, Canadá y otros, se inicio una lucha por los niños de las minorías, enfatizándose, especialmente, que los métodos tradicionales no son válidos para los ninos culturalmente diferentes y puntualizando que de acuerdo a evidencias empíricas la capacidad mental y el potencial para el aprendizaje están igualmente distribuidos en todas las razas, grupos étnicos y clases sociales.

Psicólogos y maestros que trabajan con niños en situación de desventaja encuentran que las pruebas tradicionales no revelan mucho respecto a estos niños y que los problemas que ellos presentan no son solo cognitivos y escolares, sino que son consecuencia de factores negativos interfirientes, entre los que podemos mencionar la desnutrición, la escasa estimulación o la privación socio-cultural, los cuales no permiten que se desarrolle o actualice el potencial cognitivo de estos niños.

Los problemas señalados determinaron cambios significativos en los métodos de evaluación de los niños de la minoría. Los primeros esfuerzos en este sentido se iniciaron en los Estados Unidos y culminaron en una legislación especial para defender sus derechos en la promulgación de la ley 94-142 en 1975, en la que se promovía además un cambio en el sistema de evaluación de acuerdo a las siguientes sugerencias:

1. La evaluación debe proporcionar una estimación adecuada del nivel de funcionamiento del niño dentro del contexto de su ambiente cultural y de su experiencia.

2. La evaluación debe especificar las necesidades educaciones del niño, en vez de centrarse en sus déficits intelectuales.

3. La evaluación debe estimar las habilidades del niño que sirvan de base para el desarrollo de otras habilidades. 
Orientaciones innovativas en la evaluación y educación de niños

4. La evaluación debe ser un proceso continuo y no un hecho estático.

Lo que interesaba conocer en la evaluación era lo que Vygotsky (1978) denominó la Zona de Desarrollo Próximo (ZDP), a la que definía de la siguiente forma:

La ZDP es la distancia entre el nivel de desarrollo actual del niño determinado por la solución de problemas que puede realizar en forma independiente y el nivel de desarrollo potencial determinado por la solución de problemas que puede realizar bajo la orientación de un adulto o en colaboración con compañeros más hábiles (p. 86).

Vygotsky consideraba que la evolución psicométrica tradicional concibía la inteligencia como un producto estático. Él, en cambio, la percibió como un proceso dinámico que va cambiando con el desarrollo y el aprendizaje. Para poder comprender mejor las bases conceptuales de la ZDP se revisara primero el aporte de Vygotsky respecto al origen socio-cultural de las funciones mentales superiores, y segundo, la relación entre éstas y el aprendizaje.

\section{El aporte de Vygotsky}

Este autor soviético, a través de sus estudios sobre el origen y características de las funciones psíquicas superiores, señala que estas son el resultado de la cultura y las relaciones sociales. Vygotsky (1978) concibe que en la interacción social se van construyendo las funciones psíquicas superiores -pensamiento, atención, memoria, voluntad, etc.- las que a su vez se han construido a través de la historia de la humanidad.

Otra de las premisas importantes de este autor es que el desarrollo y el aprendizaje son interdependientes. Para él no existe desarrollo sin aprendizaje, ni aprendizaje sin desarrollo. Considera que el 
aprendizaje estimula el proceso de desarrollo y éste a su vez hace posible procesos específicos de aprendizaje. Un aspecto central de su tesis es que el aprendizaje precede el desarrollo ya que éste activa los procesos evolutivos internos que operan a través de la interacción con las personas y elementos de la cultura. La instrucción se convierte así en motor y dinamizador de estos procesos. Aquí, se encuentra una de las diferencias más importantes con Piaget (1986), según el cual la educación sólo genera condiciones, facilita el desarrollo, pero no "activa o mueve" al desarrollo, manteniendo también este autor en oposición a Vigotski, que el aprendizaje está subordinado al desarrollo, es decir, que el nivel de desarrollo determina el aprendizaje.

Para el estudio de las relaciones entre aprendizaje y desarrollo, Vygotsky elabora una de sus tesis conocidas, la Zona de Desarrollo Próximo (ZDP), la cual considera que hay niveles evolutivos: el de desarrollo real y el nivel de desarrollo potencial. Considera que el nivel de desarrollo real consiste en las acciones que el niño logra realizar por sí solo, mientras que el nivel de desarrollo potencial consiste en las actividades que el niño puede realizar con ayuda de otras personas o con instrumentos mediadores externos (1978, p. 153).

Para Vygotsky, el rendimiento medido por las pruebas o por el método clínico tiene que ver con el nivel de desarrollo real, mide "conductas estáticas", conductas ya interiorizadas. En este contexto hace una fuerte crítica al psicometrismo tradicional. Sostiene que una educación centrada en la edad mental equivale a una educación centrada en el pasado de los niños. Postula que lo que interesa conocer es los que el alumno puede realizar por medio de mediadores. Para tal fin, Vygotsky considera que es importante que los educadores estimen la ZDP, la que apunta al grado de modificabilidad cognitiva. Ésta sería un indicador de las habilidades y conocimientos que pueden activarse externamente por medio del aprendizaje. Para ello el docente debe brindar experiencias de aprendizaje que amplíen el nivel de desarrollo del alumno. En este sentido, la relación, profesor- 
Orientaciones innovativas en la evaluación y educación de niños

alumno sólo puede ser vista como de cooperación. Ambos son polos activos. El alumno construye su aprendizaje con la ayuda y mediación del educador. Éste logra que los niños con niveles de desarrollo diferentes avancen en las áreas bajas. Previamente, sin embargo, el profesor tiene que conocer la inteligencia y el potencial cognitivo del niño mediante la evaluación dinámica. Dicha orientación propuesta por Vygotsky ha sido desarrollada en forma más amplia por Feuerstein cuyo enfoque lo describiremos a continuación.

\section{Contribución de Feuerstein}

Feuerstein (1979) influido por las teorías de Vygotsky, de Piaget y por sus propios trabajos con niños que aparecían con "retardo", afirma que es posible modificar las funciones cognitivas en cualquier etapa de la vida. Esta modificabilidad implica un cambio en las estructuras y lleva al educador a una "actitud modificadora activa" o enfoque activo modificante, el que conduce al niño a la corrección de sus deficiencias.

El rol del mediador es el de desarrollar los prerequisitos del pensamiento para que los alumnos puedan realizar operaciones, reflexionar, anticipar conductas, compartir significados. La preocupación más importante de Feuerstein fue la de evaluar las potencialidades del niño, en especial de los niños o adultos que habían sufrido de privación o escasa estimulación socio-cultural y tratar de remediar las deficiencias a través de un proceso de intervención activa que ayude a desarrollar una estructura cognitiva más eficaz.

Con el fin de estimar el potencial, Feuerstein utilizaba una evaluación que él denominó "Evaluación del Potencial de Aprendizaje" (EPA), la cual comenzaba con la clara definición de los objetivos: 
1. Evaluar la modificabilidad cognitiva observando el funcionamiento del niño en situaciones creadas para producir el cambio.

2. Estimar el grado de modificabilidad cognitiva.

3. Determinar la trasferencia de lo que se ha aprendido en una área a otras.

4. Identificar la modalidad de aprendizaje preferida por el niño y las estrategias para resolver problemas que sean más efectivas.

Debidamente administrada la evaluación Dinámica del Potencial de Aprendizaje (EPA) puede ser muy útil para satisfacer las necesidades y facilitar el proceso terapéutico de niños deprimidos culturalmente. Sundberg y Gonzáles (1981) han corroborado este hecho así como Martínez, Brunet y Farres (1991) y Valle y Alonso (1989) en Madrid. Todas estas investigaciones han demostrado la eficacia de estas intervenciones evidenciada en mejoras de la atención, habilidad verbal, numérica, espacial y de abstracción, así como del autoconcepto y estima de si mismo. Dichos resultados obtenidos en España han sido igualmente confirmados en Puerto Rico por Álvarez, Santos y Lebrón en 1994.

Respecto a estos resultados, Coll (1986) agrega que también hay que considerar además, el significado del aprendizaje para el niño lo que explica en los términos siguientes.

\section{César Coll y el Aprendizaje Significativo}

Coll (1986), uno de los representantes de la Psicología Genética actual (basada en la teoría piagetana y la perspectiva socio-histórica) coincide en que el aprendizaje escolar debe contribuir al desarrollo de los niños, pero señala que no basta saber cómo el docente debe trabajar las formas de pensamiento de los niños para que puedan aprender, sino que debe considerar además de las estructuras cognoscitivas el contenido. Es decir que los alumnos tienen algún co- 
nocimiento sobre los objetos o áreas del saber, además de estructuras cognoscitivas que pueden ser activadas por la información. Coll enfatiza así que resulta importante que el profesor considere que el niño ya sabe algo y que lo que sabe lo utilizará para la asimilación de contenidos nuevos. La enseñanza en la escuela debe incluir elementos que produzcan un desequilibrio o "desface óptimo" entre lo propuesto y los esquemas de conocimiento del alumno. En relación a esto, Coll sostiene que si las situaciones de aprendizaje incluyen elementos que son muy familiares para el niño, estos no le asignan significatividad, por otro lado, si son muy distantes de conocimientos previos tampoco serán significativos para el niño y el aprendizaje, si se logra, será mecánico y repetitivo. La intervención del docente entonces será crucial puesto que desde esta perspectiva tendría que acomodar la significatividad y calidad de apoyo pedagógico a los conocimientos previos de los niños y sus necesidades cambiantes.

Por tanto, siendo el alumno quien construye su aprendizaje y codifica sus esquemas de conocimiento es el docente, quien en determinados momentos, debe producir en los niños el descubrimiento de aspectos más relevantes de un tema u objeto, apoyando que los alumnos adquieran aprendizajes significativos.

\section{Conclusiones y sugerencias}

Resultados de investigaciones preliminares parecen evidenciar que la Evaluación Dinámica del Potencial de Aprendizaje es más apropiada para estimar el potencial mental de niños en situación de desventaja socio-cultural que las pruebas tradicionales como el Wechsler, Stanford - Binet, etc. Este método reduce la información y contenidos aprendidos para centrarse en la evaluación de problemas no verbales, libres del factor cultural. Incorpora también un componente de entrenamiento para ayudar al niño que no comprende la tarea. 
Esta orientación innovativa de la evaluación de la habilidad mental cambia el énfasis de puntajes cuantitativos a la observación de los procesos mentales, informa respecto a la Zona de Desarrollo Próximo (ZDP), respecto a la modalidad cognitiva preferida por el niño y respecto al efecto de la medición para cambiar las estructuras cognitivas. Se ha demostrado que esta mediación es valiosa para llenar los vacíos producidos por la privación psicocultural.

Se debe señalar, finalmente, que en nuestro medio aún no hay suficiente conocimiento de estas orientaciones innovativas en la evaluación y la educación de niños en situaciones de desventaja sociocultural. Es necesario difundir estas estrategias capacitando a maestros y dictando cursos al respecto en Facultades de Educación y Psicología.

\section{Referencias}

Álvarez V., Santos J. y Lebrón F. (1994). Efectos del programa de Enriquecimiento Instrumental de Feuerstein sobre las habilidades cognoscitivas de una muestra de estudiantes puertorriqueños. Revista latinoamericana de Psicología, 26 (1), 51-68.

Brunet, J. J. y Negro J. L. (1990). Aplicación del PEI de Feuerstein para la recuperación de alumnos con problemas de aprendizaje. En J. Martínez, J. J. Brunet y V. Farres (Eds.), Metodología de la mediación en el PEI. Madrid: Bruño.

Coll C. (1986). Psicología genética y aprendizajes escolares. México: Siglo XXI.

Coll C. (1987). Por una opción constructiva de la intervención pedagógica. En A. Álvarez (Ed.), Psicología y educación. Madrid: Bruño.

Equipo CALPA (1990). Eficacia de la aplicación del PEI en el desarrollo de las aptitudes mentales. En J. Martínez, J. J. Brunet y V. Farres (Eds.), Metodología de la mediación en el PEI. Madrid: Bruño. 
Orientaciones innovativas en la evaluación y educación de niños

Feuerstein R. (1979). The dynamic assessment of retarded performers. Baltimore: University Park Press.

Martínez J., Brunet J. J. y Farres V. (1991). Metodología de la mediación en el PEI. Madrid: Bruño.

Piaget J. (1986). Seis estudios de psicología Barcelona: Barral.

Samuda R. (1990). Assessing the abilities of minority students within a multiethnic group. Toronto: Allyn \& Bacon.

Samuda, R. (1995). Evaluation practices for the multicultural classroom. Ponencia presentada en el XXV Congreso Iberoamericano de Psicología, Puerto Rico.

Sunberg, N. y González L. (1981). Cross cultural and cross-ethnic assessment overview and issues. Advances in psychological assessment. San Francisco: Jossey Bas.

Valle, A. y Alonso E. (1989). Estudio comparativo sobre los Efectos de Aplicación del PEI en alumnos del 6to. EGB. En J. Martínez, J. J. Brunet. y V. Farres (Eds.), Metodología de la mediación en el PEI. Madrid: Bruño.

Vygotsky, L. S. (1978). Mind in society the development of higher psychological process. Nueva York: University Press. 